\title{
Predicting factors on cervical ripening and response to induction in women pregnant over 37 weeks
}

\author{
Fatemeh Bahadori $^{1}$, Hale Ayatollahi' ${ }^{1,2}$, Mohammad Naghavi-Behzad ${ }^{3}$, Hamidreza Khalkhali ${ }^{4}$, \\ Zilla Naseri ${ }^{1}$
}

${ }^{1}$ Department of Obstetrics \& Gynecology, Urmia University of Medical Sciences, Urmia, ${ }^{2}$ Mother and Infant Research Center, Urmia University of Medical Sciences, Urmia, ${ }^{3}$ Medical Philosophy and History Research Center, Tabriz University of Medical Sciences, Tabriz, ${ }^{4}$ PHD of Statistics, Urmia University of Medical Sciences, Urmia, Iran

\begin{abstract}
Induction of labor is conducted in special fetal or maternal conditions. Labor is induced in about $20 \%$ of women. The aim of this study was realizing the relationship between some factors including cervical ripening and also response to induction so we could predict the induction outcome better. Material and methods: The present prospective study was based on 101 pregnancy cases admitted to the labor ward in Urmia from March 2010 until December 2010. Maternal age ranged from 17 to 41 years and the gestational age between 37 to 42 weeks according to an ultrasound or reliable last menstrual period, as criteria of study inclusion. After admission, patients had a vaginal speculum for Preterm Premature Rupture of Membranes (PPROM), Abdominal ultrasonography for biometry and Amniotic Fluid Index (AFI), transperineal ultrasonography for measuring fetal head distance to maternal perineum, and vaginal ultrasonography for measuring cervical length and posterior angle of fetal head with cervix were undergone. Bishop score was assigned to another person. Labor was induced by administering either intravaginal misoprostol (25 microgram every six hours for a Bishop score lower than 7) or intravenous oxytocin (low dose regimen for a Bishop score equal to or more than 7). Results: Misoprostol was used for 75 patients and 26 patients had induction of labor with low dose oxytocin. Eighty one patients had Normal Vaginal Delivery (NVD) while 20 were delivered via Cesarean Section. For cervical ripening, Bishop Score $(p<0.001)$, cervical length $(p=0.04)$ and parity $(p=0.06)$ were predicting factors. The cervical posterior angle $\mathrm{p}=0.02$ had a predicting role in natural delivery. Conclusion: The cervical posterior angle was a predicting factor for natural delivery. Although cervical length, Body Mass Index (BMI), and parity were not predicting factors for natural delivery; these factors were good predictors for cervical ripening.
\end{abstract}

Keywords: successful induction, normal vaginal delivery, cesarean section

\section{Introduction}

Induction refers to the stimulation of the uterine contractions before the spontaneous onset of the labor (with or without premature rupture of membranes). Labor induction is indicated whenever the advantages for mother or fetus are more important than continuing the pregnan-

Received 26.05.2013 Accepted 31.06.2013

Med Ultrason

2013, Vol. 15, No 3, 191-198

Corresponding author: Hale Ayatollahi

Mother and Infant Research Center, Kowsar center

Motahhari Hospital

Urmia University of Medical Sciences

Urmia, Iran

Email: hayatollahi@yahoo.com cy such as rupture of membranes along with chorioamnionitis or severe pre-eclampsia $[1,2]$. Around $20 \%$ of pregnancies need induction to be terminated $[3,4]$. Pregnancy termination by induction increases the percent of caesarean sections compared with spontaneous delivery $(5,6)$ by $25 \%$ in nulliparous and $5 \%$ in multiparous cases [7-9]. It seems ideal to determine the caesarean risks before starting induction. The standard method for predicting the response to induction is based on the Bishop score for determining cervical ripening $[10,11]$. The Bishop score is defined based on scoring cervical position, cervical consistency, fatal station, cervical effacement, and cervical dilation which are all subjective criteria [12-16]. There are studies that have shown that the prediction rate of the Bishop score is weak mainly because it is a subjec- 
tive based protocol and the rate of bias is high [17-20]. In recent years, a great deal of attention has been paid to measuring the cervical length using transvaginal ultrasound as an objective method for predicting induction results. Comparisons between the two methods in predicting induction results have demonstrated great differences $[3,21]$. There are studies in which it was demonstrated that a high Body Mass Index (BMI) decreases contraction and a high dosage of oxytocin is required [22-24].

There have been many studies regarding the lack of correspondence between the prediction results in the two methods and it was observed also in the Torkildsen et al [21] and Torricelli et al [22] studies; the Park et al [23] found no significant differences. Uyar et al [13] found that in spite of the direct relationship between the cervical length and induction to delivery interval, no relationship between induction to delivery interval and Bishop score could be found [14]. Gomez et al [11] found that the cervical length was more successful than the Bishop score in predicting induction result and Eggebo et al demonstrated that the distance between the fetal head and perineum could predict induction results in the same manner as the cervical length and Bishop score. However, other authors suggest that none of the above methods are successful to predict induction results singly $[10,25,26]$.

The aim of the present study was to detect the parameter which can predict cervical ripening and successful induction in term pregnancies.

\section{Material and methods}

In this prospective study we selected 101 pregnant women using the criteria for selection used in previous studies [26-29]. Inclusion criteria were considered to be: singleton pregnancy, gestational age of 37-42 weeks (gestational dating with under 20 weeks' ultrasound or Last Menopause Period (LMP) when considered reliable), fetal membranes intact (negative Fern and Nitrazin tests), and cephalic presentation. Exclusion criteria were: non cephalic presentation, vaginal bleeding, history of surgery on uterus, placenta previa, placental abruption, onset of labor pains, multiple gestations, macrosomia, and patient's disapproval.

BMI was calculated for every patients. Ultrasound study was performed using Accuvix $-\mathrm{V}$ ten and Hoonda HS-2100 machine and fetal biometric, Amniotic Fluid Index (AFI), and diameter of the largest pocket were measured using the abdominal probe. Fetal weight was estimated by the Hadlock method [18, 28]. The distance between the fetal head to the mother's perineum was measured by an abdominal probe using a transperineal method (probe was hold transverse). The transvaginal ul-

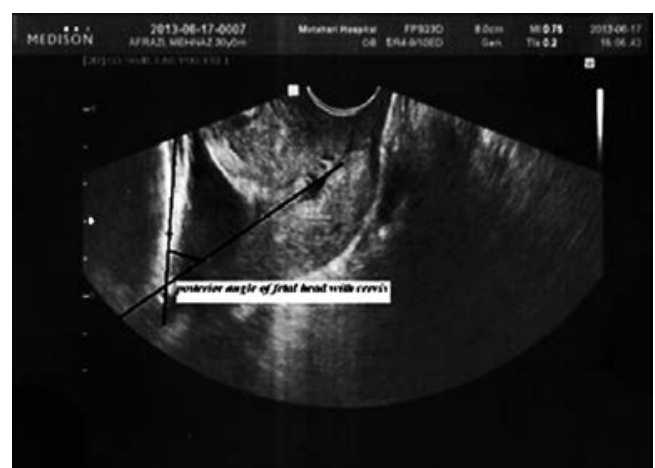

Fig 1. Posterior angle calculation during cervical length measurement

trasound was performed with an empty bladder and cervical length (interval of internal and external cervical canal) was measured and posterior cervical angle with fetal head was calculated by the same gynecologist . Posterior angle was calculated during the cervical length measurement using printed photo (fig 1). During measurement of the cervical length in order to prevent cervical deformation the minimum load over cervix was imposed. In all cases, three measurements were taken and the mean value of the three measurements was considered to be the current cervical length. The vaginal examination was conducted by another gynecologist (not informed about previous results) and the Bishop score was exactly determined based on its five parameters. Cervical ripening with misoprostol ( 25 microgram every 6 hours in posterior fornix of vagina) was considered for patients with Bishop score $\leq 6$ and induction of labor with low dose oxytocin (2 m Unit per minute) for Bishop score $\geq 7$ was considered.

Number of the required suppositories until cervical ripening, the required time from the first suppository until the cervical ripening, the induction response time and the entrance to active phase (cervical dilation more than or equal to 4 centimeter) were registered. The time interval between the beginning of the cervical ripening or induction and the natural delivery or the caesarean was also registered as well as sex and weight and the Apgar score of infant. It should be mentioned that the limitations for using suppositories were a maximum of up to 2 suppositories for cervical ripening and up to 4 suppositories ( 25 microgram every 6 hours) to enter the active phase. Induction of labor with oxytocin was up to 2 times, if the active phase was not established within 48 hours after entering the study.

All informations were collected by a checklist and finally by using SPSS 16.0 software, qualitative variables were analyzed by the chi-square statistical test and quantitative variables were analyzed by the t-test. $\mathrm{P} \leq 0.05$ was consid- 
ered to be significant to determine the relationship between factors and cervical ripening and response induction.

Patients were free to leave the study and privacy policy was considered for patients. All stages of the study were verified by the Ethical Committee of the Urmia University of Medical Sciences which was in accordance with the Declaration of Helsinki.

\section{Results}

Study group demographic and clinical characteristics are summarized in Table I

The reasons for caesarean were fetal distress in 9 cases $(45 \%)$, ceohalopelvic disproportion (CPD) in 6 cases $(30 \%)$, placental abruption in 3 cases $(15 \%)$, and meconium staining of amniotic fluid in 2 cases $(10 \%)$.

Patients were categorized based on their parity as: nulliparous 58.4\%)(59 patients) , vaginal delivery 19.8\% (20 patients), two vaginal deliveries $11.9 \%$ (12 patients), three vaginal deliveries 5.9\% (6 patients), four vaginal deliveries $3 \%$ (3 patients), and five vaginal deliveries $1 \%$ (one patients).

The reasons for the hospitalization of patients under the study have been shown in Table II.

Among 101 patients, 75 (74.3\%) had the Bishop score $\leq 6$ and in these patients E1 (misoprostol intravaginal) was used at the beginning of intervention for their cervical ripening. In the remaining 26 patients $(25.7 \%)$ with Bishop score $\leq 7$ induction was started by oxytocin $2 \mathrm{~m}$ Unit per minute. Patients who reached Bishop score $\leq 7$ during 12 hours were considered to be successful in cervical ripening and those with a Bishop score less than 7 after 12 hours of intervention were considered the failed group in cervical ripening. The two groups were compared and the results are summarized in table III.
Table I. Clinical and demographic factors in the study population

\begin{tabular}{lc}
\hline Variant & Mean \pm SD \\
\hline Age (years) & $26.7 \pm 6.35$ \\
BMI (kg/m2) & $29.8 \pm 2.74$ \\
Pregnancy age (days) & $278.42 \pm 6.35$ \\
Parity & $1.1 \pm 0.78$ \\
Bishop score & $4.4 \pm 2.5$ \\
Cervix length (cm) & $2.93 \pm 0.82$ \\
Amniotic liquid index & $8.98 \pm 3.51$ \\
Posterior cervical angle & $109.7 \pm 16.45$ \\
Estimating fetal weight (gr) & $3470 \pm 333.3$ \\
Fetal head to perineum distance (cm2) & $6.54 \pm 0.99$ \\
Time from including to study till cervical & $452.2 \pm 505.8$ \\
ripening (min) & \\
Time from entrance to active phase delivery & $608.56 \pm 7.37$ \\
(min) & $639.27 \pm 9.33$ \\
Time from entrance to delivery (min) & $3470 \pm 333.3$ \\
\hline Estimating fetal weight (gr) & \\
\hline
\end{tabular}

BMI: Body Mass Index

Table II. Hospitalization reasons in the study group

\begin{tabular}{lc}
\hline Reason of Hospitalization & Frequency: number (\%) \\
\hline Reduced fetal movement & $37(36.6)$ \\
Postdate & $16(15.8)$ \\
Oligohydramnios & $15(14.9)$ \\
Hypertension & $14(13.9)$ \\
Low biophysical profile & $5(5)$ \\
Thrombocytopenia & $3(3)$ \\
Proteinuria & $3(3)$ \\
\hline
\end{tabular}

Table III. Comparing means of factors with success in cervical ripening (12 hours)

\begin{tabular}{lccc}
\hline \multirow{2}{*}{ Factors } & \multicolumn{2}{c}{$\begin{array}{c}\text { Mean } \pm \text { Standard Deviation } \\
\text { Unsuccessful cervical ripening (N=21) }\end{array}$} \\
\hline Age (year) & Successful cervical ripening (N=54) & $25.91 \pm 6.12$ & 0.37 \\
Gestational Age (day) & $27.29 \pm 9.06$ & $277.86 \pm 7.62$ & 0.63 \\
Gravidity & $278.80 \pm 7.76$ & $1.50 \pm 0.9$ & 0.07 \\
Parity & $2.04 \pm 1.2$ & $0.32 \pm 0.56$ & $\mathbf{0 . 0 2}$ \\
BMI & $0.95 \pm 1.2$ & $30.74 \pm 2.97$ & 0.19 \\
Bishop score (cervix score) & $29.82 \pm 2.73$ & $1.86 \pm 1.69$ & $\mathbf{0 . 0 0 1}$ \\
Cervix length (cm) & $3.72 \pm 1.72$ & $3.37 \pm 0.84$ \\
Fetal head and mother's perineum distance & $2.96 \pm 0.79$ & $6.77 \pm 0.82$ \\
Posterior cervical angle & $6.51 \pm 1.07$ & $106.68 \pm 14.38$ \\
Estimated fatal weight & $106.33 \pm 14.91$ & $3453.55 \pm 313.56$ \\
Amniotic fluid index & $3443.98 \pm 356.17$ & $9.19 \pm 3.06$ \\
Deepest Pocket of amniotic fluid & $8.96 \pm 3.80$ & $3.27 \pm 1.51$ & 0.3 \\
\hline
\end{tabular}


194 Fatemeh Bahadori et al

Predicting factors on cervical ripening and response to induction in women pregnant over 37 weeks

Table IV. Characteristics of the study patients assigned in successful and unsuccessful response to induction groups

\begin{tabular}{lccc}
\hline \multirow{2}{*}{ Factors } & \multicolumn{2}{c}{ Mean \pm Standard Deviation } \\
& Successful induction (N=90) & Unsuccessful induction (N=11) \\
\hline Age (year) & $276.9 \pm 18.20$ & $25.91 \pm 6.12$ & 0.61 \\
Gestational Age (day) & $278.60 \pm 7.42$ & $277.86 \pm 7.62$ & 0.48 \\
Gravidity & $1.96 \pm 1.29$ & $1.50 \pm 0.9$ & 0.43 \\
Parity & $0.82 \pm 1.19$ & $0.32 \pm 0.56$ & 0.32 \\
BMI & $29.64 \pm 2.29$ & $30.74 \pm 2.97$ & $\mathbf{0 . 0 4}$ \\
Bishop score (cervix score) & $4.70 \pm 2.48$ & $3.86 \pm 1.69$ & $\mathbf{0 . 0 0 4}$ \\
Cervix length (cm) & $2.88 \pm 0.83$ & $6.77 \pm 0.82$ & 0.06 \\
Fetal head - mother's perineum distance & $6.52 \pm 1.01$ & $106.68 \pm 14.38$ \\
Posterior cervical angle & $110.23 \pm 17.07$ & $3453.55 \pm 313.56$ \\
Estimated fatal weight & $3468.89 \pm 332.78$ & $9.19 \pm 3.06$ \\
Amniotic fluid index & $8.92 \pm 3.59$ & $3.27 \pm 1.51$ \\
Deepest pocket of amniotic fluid & $3.25 \pm 1.42$ & 0.52 \\
\hline
\end{tabular}

Table V. Characteristics of the study patients assigned depending on the delivery type

\begin{tabular}{lccc}
\hline Factors & \multicolumn{2}{c}{ Mean \pm Standard Deviation } & Cesarean (N=21) \\
\hline Age (year) & Natural (N=80) & $26.58 \pm 5.39$ & 0.93 \\
Gestational Age (day) & $26.69 \pm 6.66$ & $278.73 \pm 7.41$ & 0.92 \\
Gravidity & $278.58 \pm 7.50$ & $1.69 \pm 0.97$ & 0.33 \\
Parity & $1.96 \pm 1.31$ & $0.98 \pm 0.62$ & 0.43 \\
BMI & $1.18 \pm 0.81$ & $30.24 \pm 2.72$ & 0.42 \\
Bishop score (cervix score) & $29.75 \pm 2.68$ & $3.61 \pm 2.66$ & 0.11 \\
Cervix length & $4.55 \pm 2.60$ & $3.15 \pm 0.84$ & 0.16 \\
Fetal head - mother's perineum distance & $2.89 \pm 0.81$ & $6.65 \pm 0.87$ & 0.64 \\
Posterior cervical angle & $6.55 \pm 1.01$ & $98.23 \pm 14.02$ & $\mathbf{0 . 0 0 1}$ \\
Estimated fatal weight & $112.57 \pm 15.90$ & $3562.35 \pm 349.61$ & 0.16 \\
Amniotic fluid index & $3456.89 \pm 330.83$ & $10.02 \pm 4.01$ & 0.06 \\
Deepest pocket of amniotic fluid & $8.55 \pm 3.24$ & $3.63 \pm 1.68$ & 0.08 \\
\hline
\end{tabular}

Table VI. The relationships between measured variables and predicting response to induction in pregnant women with delivery occurring during the first 24 hours

\begin{tabular}{lccc}
\hline Factors & \multicolumn{2}{c}{ Mean \pm Standard Deviation } & P \\
& Successful natural delivery (N=69) & Unsuccessful natural delivery (N=12) & 0.53 \\
\hline Age (year) & $26.88 \pm 6.72$ & $25.56 \pm 6.14$ & 0.28 \\
Gestational age (day) & $279.10 \pm 7.13$ & $276.58 \pm 8.90$ & 0.18 \\
Gravidity & $2.04 \pm 1.37$ & $1.50 \pm 0.79$ & 0.009 \\
Parity & $0.90 \pm 1.25$ & $0.33 \pm 0.49$ & 0.23 \\
BMI & $29.60 \pm 2.62$ & $30.60 \pm 2.95$ & 0.001 \\
Bishop score (cervix score) & $4.94 \pm 2.51$ & $2.33 \pm 1.92$ & 0.003 \\
Cervix length & $2.78 \pm 0.78$ & $3.52 \pm 0.67$ & 0.38 \\
Fetal head - mother's perineum distance & $6.51 \pm 1.03$ & $6.76 \pm 0.87$ & 0.89 \\
Posterior cervical angle & $112.67 \pm 16.60$ & $112 \pm 11.60$ & $3524 \pm 380.74$ \\
Estimated fatal weight & $3445.22 \pm 339.23$ & $9.44 \pm 3.80$ & 0.45 \\
Amniotic fluid Index & $8.40 \pm 3.14$ & $3.52 \pm 1.73$ & 0.19 \\
Deepest pocket of amniotic fluid & $3.03 \pm 1.11$ & &
\end{tabular}


Table VII. The relationships between measured variables and predicting response to induction in pregnant women with delivery occurring during the first 36 hours

\begin{tabular}{lccc}
\hline Factors & \multicolumn{2}{c}{$\begin{array}{c}\text { Mean } \pm \text { Standard Deviation } \\
\text { Unsuccessful natural delivery (N=7) }\end{array}$} \\
\hline Age (year) & $26.93 \pm 6.65$ & $24.14 \pm 6.69$ & 0.29 \\
Gestational age (day) & $278.93 \pm 7.37$ & $276.57 \pm 8.05$ & 0.42 \\
Gravidity & $2.01 \pm 1.63$ & $1.43 \pm 0.78$ & 0.26 \\
Parity & $0.86 \pm 1.22$ & $0.29 \pm 0.48$ & 0.02 \\
BMI & $29.68 \pm 2.62$ & $30.42 \pm 3.43$ & 0.49 \\
Bishop score (cervix score) & $4.74 \pm 2.62$ & $2.57 \pm 1.13$ & 0.001 \\
Cervix length & $2.85 \pm 0.82$ & $3.37 \pm 0.53$ & 0.04 \\
Fetal head - mother's perineum distance & $6.56 \pm 1.03$ & $6.47 \pm 0.78$ & 0.82 \\
Posterior cervical angle & $112.64 \pm 16.45$ & $111.86 \pm 8.76$ \\
Estimated fatal weight & $3453.23 \pm 336.57$ & $3495.57 \pm 281.37$ \\
Amniotic fluid Index & $8.66 \pm 3.30$ & $7.37 \pm 2.40$ & 0.90 \\
Deepest pocket of amniotic fluid & $3.15 \pm 1.25$ & $2.54 \pm 0.58$ & 0.74 \\
\hline
\end{tabular}

ROC Curve

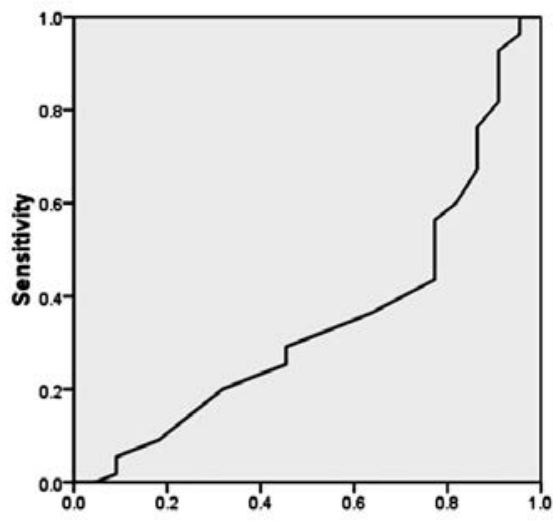

Fig 2. ROC curve for determining cut off point related to cervical length

Irrespective of the Bishop score the patients with cervical dilation $\geq 4 \mathrm{~cm}$ in the first 24 hours were considered to be the group with a successful response to induction (90 patients). An unsuccessful induction was obtained in 11 patients. Characteristics of the study patients assigned in successful and unsuccessful response to induction groups are summarized in table IV. Characteristics of the study patients assigned depending on the delivery type (natural- 80 patients and cesarean- 21 patients) can be found in table V.

The relationships between measured variables and predicting response to induction in pregnant women depending on the occurrence of delivery (during the first 24 hours, and also during the first 36 hours) are analyzed in table VI and table VII respectively.
ROC curve was used in order to determine the cutoff point related to the cervical length (fig 2): $2.2 \mathrm{~cm}$ for cervical length has a sensitivity of $82 \%$ and specificity of $91 \%$ and could be used as a proper cut off point for cervical length.

\section{Discussion}

In various clinical cases it is necessary to terminate pregnancy [26]. Predicting induction success is one of the important and challenging issues for the doctor. So it is important to investigate the factors affecting it. One of the most important methods for predicting the response to induction is the Bishop score which has been used for a long timenow. As our study showed, the role of the Bishop score in predicting cervical ripening and response to induction, was more powerful than other factors.

In the study by Uyar et al, cervical length and BMI had a positive relationship with interval induction to the delivery time interval, while dilation and effacement had a negative relationship with induction to the delivery time interval [13]. No relationship between induction to delivery time interval on one hand and Bishop score, parity, age, and neonatal weight in the other hand was found and the value of cervical length was more helpful than other factors in predicting induction [14]. In our study the Bishop score was more powerful than other factors in predicting cervical ripening and response to induction.

In the study of Gomez et al the significance of the transvaginal cervical length measurement and the Bishop score calculation in predicting delivery induction was in- 
vestigated. In this study the cervical length had priority on the Bishop score in predicting the success of induction and had sensitivity and specificity of $66 \%$ and $76 \%$ compared with $77 \%$ and $56 \%$ for the Bishop score [12]. In our study the role of the Bishop score was more significant than other factors in cervical ripening and response to induction.

In the study of Meijer-Hoogeveen et al it was found that the cervical length in standing or prone positions is a stronger factor in predicting unsuccessful induction [13], rather than the Bishop score, which is contrary to our results.

In the Jamal et al study the Bishop score showed a linear relation with the latent phase time and the induction to delivery time. Women with cervical length $<2.6$ $\mathrm{cm}$ and Bishop score $>3$ had a shorter latent phase with higher probability for delivery and both factors predict duration and type of delivery [10]. These results are in accordance with that of ours.

Of course the important point is that the Bishop score did not have a significant relationship with vaginal delivery which is our final goal, so it is necessary to start ripening and induction even in low Bishop score cases.

In our study, the cervical length gained a more important role after neutralizing the role of the Bishop score and a cut off was determined equal to $2.2 \mathrm{~cm}$ based on the ROC curve with a sensitivity of $82 \%$ and specificity of $91 \%$, compared with the cut off estimated at $>2.6$ by Jamal et al [10].

In a study by Eggebo et al predicting response to delivery induction was conducted through the ultrasonographic measurement of the distance between the fetal head and mother's perineum and comparing it with other maternal and fetal factors. In spite of our results, the distance between the fetal head and perineum could predict a result similar to the cervical length and Bishop score, but the author claimed that none of the findings above are successful individually in predicting induction results and further studies would show us a combination of these factors [25].

In a study by Nikbakht et al, the cervical length was compared with that of the Bishop score for predicting a type of delivery [26]. Findings showed that sensitivity levels of the cervical length test and the Bishop score in the true predicting of natural delivery are $71.6 \%$ and $53.4 \%$ respectively and the specificity in diagnosing caesarean are $45 \%$ and $66.7 \%$ respectively. This study concluded that there is no difference between measuring the cervical length with an index of 27 millimeters and the Bishop score of 4 in predicting the method of pregnancy termination.
We found a significant relationship between the parity and cervical ripening in our study. Similar results were obtained by Eggebo et al [25] and Nikbakht et al[27].

Also similar to the study by Park et al. there is a significant relationship between reaching the active phase and BMI which was equal to the Bishop score in our study. However, in our study this relationship could not be observed in predicting the cervical ripening [30].

We found no significant relationship between the fetal head to the perineum distance and cervical ripening or reaching active phase or vaginal delivery contrary to the results of Eggebo et al [24]. We think that ethnical and regional differences are responsible for this difference.

No significant relationship between the variable of fetal weight estimation and the cervical ripening amount or reaching the active phase or delivery was obtained. Also, no significant relationship between variables of AFI, mother's age, and gestational age on one hand and cervical ripening index or reaching active phase or delivery on the other hand was observed in our study.

Among 81 cases of natural delivery, only one case had a delivery in more than 48 hours, which shows better response of pregnant women in our region comparing with that of women included in the study of Gomez et al [11]. In the group with natural delivery during the first 24 hours, the natural delivery had a significant relationship with the Bishop score, cervical length, and parity. The same correlations were obtained in the group with natural delivery in the first 36 hours. AFI with the deepest pocket of amniotic fluid had a significant relationship with natural delivery in suspected cases. More investigations in this field are needed.

Finally, the interesting finding of our study was that in spite of the strong significant relationship between the Bishop score in cervical ripening and also reaching the active phase, there was no significant relationship between the Bishop score and occurrence of natural delivery. Also, despite the lack of a significant relationship between the posterior cervical angle and cervical ripening and reaching active phase, the posterior cervical angle had a significant relationship with the occurrence of a natural delivery, similar to Eggebo et al results [25].

\section{Limits of the study}

Beside all the results which were found in this study, there were just few probable factors which have to be taken into account, Also this study would have been more reliable if it was conducted on a larger population.

\section{Conclusions}

The cervical posterior angle could be considered a predicting factor for natural delivery. The cervical length, 
BMI, and parity are not predicting factors for natural delivery although these factors are good predictors for cervical ripening. Our study shows that predicting cervical ripening or reaching active phase could be different from predicting the final vaginal delivery. On the other hand regarding the lack of routine measurement of the posterior angle for predicting the delivery process, it would be possible to propose a solution for predicting final delivery results by continuing this study and conducting supplementary researches.

\section{Conflict of interest: none}

\section{References}

1. DiSaia PJ, Creasman WT. Clinical Gynecologic Oncology: Expert Consult-Online: Saunders; 2012.

2. Mandruzzato G, Alfirevic Z, Chervenak F, et al. Guidelines for the management of postterm pregnancy. J Perinat Med 2010; 38: 111-119.

3. Pandis GK, Papageorghiou AT, Ramanathan VG, Thompson $\mathrm{MO}$, Nicolaides KH. Preinduction sonographic measurement of cervical length in the prediction of successful induction of labor. Ultrasound Obstet Gynecol 2001;18:623-628.

4. Baacke KA, Edwards RK. Preinduction cervical assessment. Clin Obstet Gynecol 2006; 49: 564-572.

5. Vahratian A, Zhang J, Troendle JF, Sciscione AC, Hoffman MK. Labor progression and risk of cesarean delivery in electively induced nulliparas. Obstet Gynecol 2005; 105: 698-704.

6. Smith JG, Merrill DC. Oxytocin for induction of labor. ClinObstet Gynecol 2006; 49: 594-608.

7. Heffner LJ, Elkin E, Fretts RC. Impact of labor induction, gestational age, and maternal age on cesarean delivery rates. Obstet Gynecol 2003; 102: 287-293.

8. Caughey AB, Nicholson JM, Cheng YW, Lyell DJ, Washington AE. Induction of labor and cesarean delivery by gestational age. Am J Obstet Gynecol 2006; 195: 700-705.

9. Taghavi S, Barband S, Alikhah H, Bagheri-Asl M, Naghavi-Behzad M. Outcome of Pregnancies Presenting for Diagnostic Evaluation of Fetal Trisomy. Life Sci J 2013; 10: 1682-1687.

10. Jamal A, Rezasoltani R, Marsoosi V. OP22.01: Comparison of Bishop score and transvaginal ultrasound assessment of the cervix in prediction of labor induction. Ultrasound Obstet Gynecol 2007; 30: 532.

11. Kaufman KE, Bailit JL, Grobman W. Elective induction: an analysis of economic and health consequences. Am J Obstet Gynecol 2002; 187: 858-863.

12. Gomez Laencina AM, Sanchez FG, Gimenez JH, Martinez MS, Valverde Martínez JA, Vizcaíno VM. Comparison of ultrasonographic cervical length and the Bishop score in predicting successful labor induction. Acta Obstet Gynecol Scand 2007; 86: 799-804.
13. Meijer-Hoogeveen M, Roos C, Arabin B, Stoutenbeek P, Visser G. Transvaginal ultrasound measurement of cervical length in the supine and upright positions versus Bishop score in predicting successful induction of labor at term. Ultrasound Obstet Gynecol 2009; 33: 213-220.

14. Uyar Y, Erbay G, Demir BC, Baytur Y. Comparison of the Bishop score, body mass index and transvaginal cervical length in predicting the success of labor induction. Arch Gynecol Obstet 2009;280:357-362.

15. Sehati-Shafaii F, Norouzi-Panahi L, Piri R, Naghavi-Behzad M, Naghi-Zadeh S. Comparative analysis of pregnancy outcome in pregnant women in active and latent phase of pregnancy; A Study from a Referral Center in Northwestern Iran. Life Sci J 2013;10:2095-2101.

16. Ghojazadeh M, Naghavi-Behzad M, Azar ZF, Saleh P, Ghorashi S, Pouri AA. Parental Knowledge and Attitudes about Human Papilloma Virus in Iran. Asian Pac J Cancer Prev 2012; 13: 6169-6173.

17. Levy R, Zaks S, Ben-Arie A, Perlman S, Hagay Z, Vaisbuch E. Can angle of progression in pregnant women before onset of labor predict mode of delivery? Ultrasound Obstet Gynecol 2012; 40: 332-337.

18. Rane SM, Guirgis RR, Higgins B, Nicolaides K. Models for the prediction of successful induction of labor based on pre-induction sonographic measurement of cervical length. J Matern Fetal Neonatal Med 2005; 17: 315-322.

19. Ross MG, Beall MH, editors. Prediction of preterm birth: nonsonographic cervical methods. Seminars in perinatology. Elsevier; 2009.

20. Ghojazadeh M, Azar ZF, Saleh P, Naghavi-Behzad M, Azar NG. Knowledge and Attitude of Iranian University Students toward Human Papilloma Virus. Asian Pac J Cancer Prev 2012; 13: 6115-6119.

21. Park KH. Transvaginal ultrasonographic cervical measurement in predicting failed labor induction and cesarean delivery for failure to progress in nulliparous women. J Korean Med Sci 2007; 22: 722-727.

22. Pevzner L, Powers BL, Rayburn WF, Rumney P, Wing DA. Effects of maternal obesity on duration and outcomes of prostaglandin cervical ripening and labor induction. Obstet Gynecol 2009; 114: 1315-1321.

23. Zhang J, Bricker L, Wray S, Quenby S. Poor uterine contractility in obese women. BJOG 2007; 114: 343-348.

24. Poobalan AS, Aucott LS, Gurung T, Smith WC, Bhattacharya $\mathrm{S}$. Obesity as an independent risk factor for elective and emergency caesarean delivery in nulliparous women-systematic review and meta-analysis of cohort studies. Obes Rev 2009; 10: 28-35.

25. Eggebo TM, Okland I, Heien C, Gjessing LK, Romundstad $\mathrm{P}$, Salvesen KA. Can ultrasound measurements replace digitally assessed elements of the Bishop score? Acta Obstet Gynecol Scand 2009; 88: 325-331.

26. Crane JM. Factors predicting labor induction success: a critical analysis. Clin Obstet Gynecol 2006; 49: 573584.

27. Nikbakht R, Saharkhiz N, Pour sayah N. Comparison of Cervical Length Measurement by Transvaginal Ultra- 
sonograghy and Bishop Score in Predicting Successful Labor Induction. Jundishapur Sci Med J 2010; 9.

28. Rane SM, Guirgis RR, Higgins B, Nicolaides KH. The value of ultrasound in the prediction of successful induction of labor. Ultrasound Obstet Gynecol 2004; 24: 538-549.

29. Vrouenraets FP, Roumen FJ, Dehing CJ, van den Akker ES, Aarts MJ, Scheve EJ. Bishop score and risk of cesarean de- livery after induction of labor in nulliparous women. Obstet Gynecol 2005; 105: 690-697.

30. Park KH, Hong JS, Kang WS, Shin DM, Kim SN. Body mass index, Bishop score, and sonographic measurement of the cervical length as predictors of successful labor induction in twin gestations. J Perinat Med 2009; 37: 519-523. 